\title{
Enhancement of Pathogen Resistance by Activation of Pattern Recognition Receptors (PRR) Mediated Immunity
}

ISSN: 2637-7659

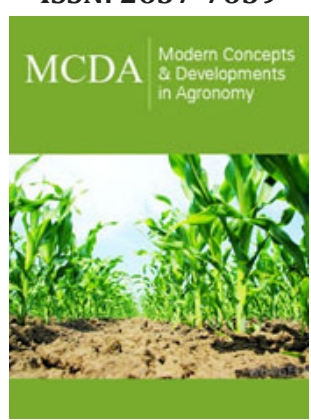

*Corresponding author: Lorena Pizarro, Agrifood, Animal and Environmental Sciences (ICA3), University of O'Higgins, Chile

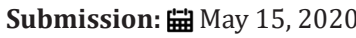

Published: 海June 03, 2020

Volume 6 - Issue 3

How to cite this article: Lorena Pizarro, Adi Avni. Enhancement of Pathogen Resistance by Activation of Pattern Recognition Receptors (Prr) Mediated Immunity. Mod Concep Dev Agrono. 6(3). MCDA. 000638. 2020. DOI: 10.31031/MCDA.2020.06.000638

Copyright@ Lorena Pizarro, This article is distributed under the terms of the Creative Commons Attribution 4.0 International License, which permits unrestricted use and redistribution provided that the original author and source are credited.

\author{
Lorena Pizarro ${ }^{1 *}$ and Adi Avni ${ }^{2}$ \\ ${ }^{1}$ Agrifood, Animal and Environmental Sciences (ICA3), University of O'Higgins, Chile \\ ${ }^{2}$ School of Plant Sciences and Food Security, Tel Aviv University, Israel
}

\begin{abstract}
Modern agriculture control diseases by extensive application of chemicals, however, this strategy brings undesired effects, such as environmental contamination and development of pesticide-resistant pathogens. Therefore, the need to develop alternative or supplementary strategies to control crop diseases has arisen. Plant immunity relies on the capacity of each cell to recognize pathogen threat, through cell surface and intracellular receptors, initiating immune responses. Cell surface receptors are called Pattern Recognition Receptors (PRRs) by their ability to recognizing characteristic microbial-derived molecules called microbe-associated molecular patterns (MAMPs). After PRR activation defence responses are elicited enabling the plant to ward off pathogen colonization. MAMPs are distinctive molecules present in bacteria or fungi, non-strain specific, such as bacterial flagellin and fungal endo-xylanases, which are recognized by specific PRRs, forming the MAMP/PRR recognition system that triggers the defence. Recent evidence shows that after MAMP exposure plant acquire resistance to pathogens, highlighting the potential of exploiting PRR activation for enhancing pathogen resistance crops fields.
\end{abstract}

Keywords: PRR; Elicitors; Plant immunity; MAMP/PRR recognition system

Abbreviations: SAR: Systemic Acquire Resistance; PRR: Pattern Recognition Receptors; MAMP: Microbial Associated Molecular Pattern

\section{Introduction}

Currently, agriculture practices control pathogen diseases by application of chemical pesticides in fields, but these technique causes environmental contamination and in some cases human health risks. Moreover, high pressure of pesticides leads to loss of effectiveness of these products due to the development of resistance mechanisms of pathogens [1,2]. In this scenario surge the need to develop complementary or alternative strategies for pest control in fields. An emerging option is to activate plant immunity with chemical or natural inducers, also known as immune activators. Immune activators have not antimicrobial or fungicide activity, the action mode is based on activation of plant immunity, typically by systemic acquired resistance (SAR) pathways [3]. The agroindustry has developed natural and chemical immune-activators and hormone analogues to prime or enhance plant defence response.

Plants have an inducible innate immune system to cope with microorganism attacks. Plant immunity relies on the recognition of pathogen-derived molecules to triger innate immunity. Plant plasma membrane receptors, called Pattern Recognition Receptors (PRRs) recognize distinctive microorganism molecules, named Microbe-Associated Molecular Patterns (MAMPs), present in bacteria or fungi, in a non-strain specific manner, such as lipooligosaccharides of gram-negative bacteria, bacterial flagellin, bacterial Elongation Factor$\mathrm{Tu}(\mathrm{EF}-\mathrm{Tu})$, glucans and glycoproteins from oomycetes, chitin and endo-xylanases from fungi, among others [4]. These MAMPs are recognized by specific PRRs, forming the MAMP/PRR recognition system, which activates a signalling cascade, that leads to a robust transcriptional and physiological response to hamper pathogen colonization (Figure 1) [4]. Through MAMP/ PRR recognition system, plants can sense and defend themselves against pathogens from different races and species that share a MAMP, making PRR mediated immunity a versatile and efficient defence system. 


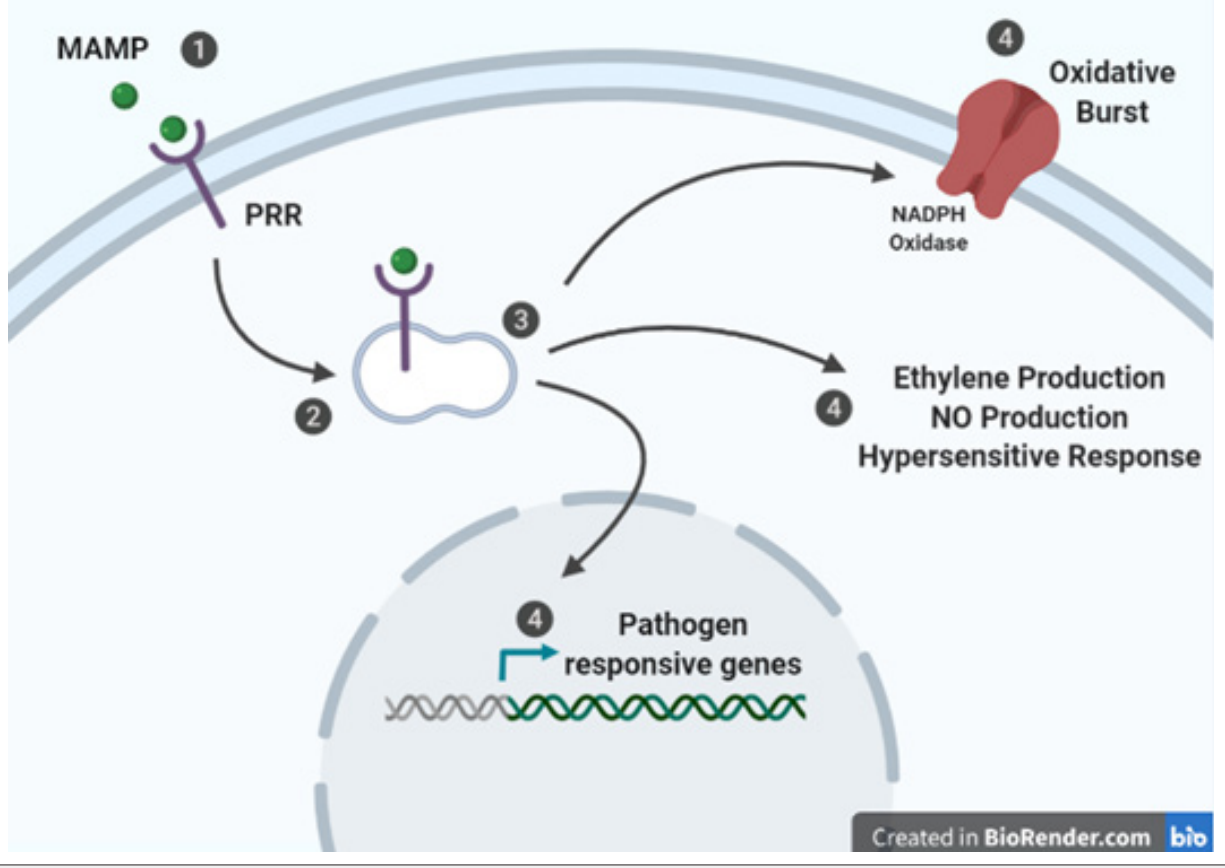

Figure 1: MAMP/PRR recognition system model. Step 1: MAMP is recognized by PRR.

Step 2: PRR is internalized by endocytosis after MAMP recognition. Step 3: Signaling.

Step 4: Activation of Defense Responses.

A scheme designed using biorender ${ }^{\circledR}$

\section{Discussion}

\section{Activation of PRR for enhancing plant resistance}

By the application of MAMPs plant immunity is activated, triggering defence responses that restrict pathogen attack, therefore MAMPs could be used as immune activators for inducing plant immunity and pathogen resistance [3,5]. Two well-described MAMP/PRR recognition model systems are flg22/ FLS2 and EIX/LeEIX2. Flg22/FLS2 recognition system is formed by an RLK-type PRR that recognizes flagellin, one of the main proteins composing bacterial flagella, from diverse bacteria such as Bacillus sp, Pseudomonas sp and Xanthomonas sp [6-8]. While EIX/LeEIX2 recognition system is formed by an RLP-type PRR that recognize a fungal endo-xylanase, secreted by diverse fungi such as Trichoderma viridea and Botrytis cinerea [9-12]. In Arabidopsis and tomato exposure to flg22 confers resistance to the bacteria Pseudomonas sp, fungus Fusarium sp, and even against the virus GBNV [13-15]. While exposure to EIX-like endo-xylanase enhances resistance against B. cinerea and the virus TMV in tobacco [16]. This evidence presents the induction of plant immunity mediated by PRR activation as a powerful approach for enhancing pathogen resistance, and for this as a possible alternative strategy for disease control in fields.

\section{Conclusion}

The utilization of MAMP/PRR signalling mechanism is a compelling approach for developing agricultural strategies to ward off possible outbreaks of disease and in the development of environmentally friendly disease control strategies. Then, it is opportune to explore deep in this area to promote and develop new alternative or complementary procedures for disease management in crop fields.

\section{Acknowledgement}

This work was partially supported by CONICYT+PAI/ Convocatoria Nacional Subvención a la Instalación en la Academia, Convocatorio 2019 Folio PAI 77190027 (LP) and Israel Science Foundation administered by the Israel Academy of Science and Humanities (550/18) (AA).

\section{Conflict of Interest}

The authors declare that the research was conducted in the absence of any commercial or financial relationships that could be construed as a potential conflict of interest.

\section{References}

1. Mikaberidze A, Paveley N, Bonhoeffer S, van den Bosch F (2017) Emergence of resistance to fungicides: The role of fungicide dose. Phytopathology 107(5): 545-560.

2. Sundin GW, Wang N (2018) Antibiotic resistance in plant-pathogenic bacteria. Annu Rev Phytopathol 56: 161-180.

3. Conrath U, GJM Beckers, CJG Langenbach, MR Jaskiewicz (2015) Priming for enhanced defense. Annual Review of Phytopathology 53: 97-119.

4. Macho AP, Zipfel N (2014) Plant PRRs and the activation of innate immune signaling. Mol Cell 54(2): 263-272. 
5. Burketova L, Trda L, Ott PG, Valentova O (2015) Bio-based resistance inducers for sustainable plant protection against pathogens. Biotechnol Adv 33(6): 994-1004.

6. Ren JJ, Shi GL, Wang XQ Liu JG, Wang YN (2013) Identification and characterization of a novel Bacillus subtilis strain with potent antifungal activity of a flagellin-like protein. World J Microb Biot 29(12): 23432352 .

7. Sun WX, Dunning FM, Pfund C, Weingarten R, Bent AF (2006) Withinspecies flagellin polymorphism in Xanthomonas Campestris Pv Campestris and its impact on elicitation of Arabidopsis FLAGELLIN SENSING2-dependent defenses. Plant Cell 18(3): 764-779.

8. Zeng WQ, He SY (2010) A prominent role of the flagellin receptor FLAGELLIN-SENSING2 in mediating stomatal response to pseudomonas syringae pv tomato DC3000 in arabidopsis. Plant Physiol 153(3): 11881198.

9. Avni A, Bailey BA, Mattoo AK, Anderson JD (1994) Induction of ethylene biosynthesis in nicotiana-tabacum by a trichoderma-viride xylanase is correlated to the accumulation of 1-Aminocyclopropane-1-Carboxylic Acid (Acc) synthase and Acc Oxidase Transcripts. Plant Physiol 106(3): 1049-1055.

10. Frias M, Gonzalez M, Gonzalez C, Brito N (2019) A 25-Residue peptide from Botrytis cinerea Xylanase BcXyn11A Elicits Plant Defenses. Front Plant Sci 10: 474
11. Noda J, Brito N, Gonzalez C (2010) The Botrytis cinerea xylanase Xyn11A contributes to virulence with its necrotizing activity, not with its catalytic activity. BMC Plant Biol 10: 38.

12. Ron M, Avni A (2004) The receptor for the fungal elicitor ethyleneinducing xylanase is a member of a resistance-like gene family in tomato. Plant Cell 16(6): 1604-1615.

13. Mishina TE, Zeier J (2007) Pathogen-associated molecular pattern recognition rather than development of tissue necrosis contributes to bacterial induction of systemic acquired resistance in Arabidopsis. Plant J 50(3): 500-513.

14. Sarowar S, Alam ST, Makandar R, Lee H, Trick HN, et al. (2019) Targeting the pattern-triggered immunity pathway to enhance resistance to Fusarium graminearum. Mol Plant Pathol 20(5): 626-640.

15. Vanthana M, Nakkeeran S, Malathi VG, Renukadevi P, Vinodkumar S (2019) Induction of in planta resistance by flagellin (Fig) and elongation factor-TU (EF-Tu) of Bacillus amyloliquefaciens (VB7) against groundnut bud necrosis virus in tomato. Microb Pathogenesis 137.

16. Yang YK, Yang XF, Dong YJ, Qiu DW (2018) The Botrytis cinerea Xylanase BcXyl1 modulates plant immunity. Front Microbiol 9: 2535. 